\title{
Análise de sobrevivência e estimativa de entropia para Sarconesia chlorogaster (Wiedemann) (Diptera, Calliphoridae) $^{1}$
}

\author{
Mauricio O. Moura ${ }^{2}$ \\ Sionei R. Bonatto ${ }^{2}$
}

\begin{abstract}
Survival analysis and estimation of entropy of Sarconesia chlorogaster (Wiedemann) (Diptera, Calliphoridae). The life expectancy of Sarconesia chlorogaster (Wiedemann, 1830) reared in artificial diet and controlled temperatures chamber set at $27 \pm 1^{\circ} \mathrm{C}, 70 \pm 10 \% \mathrm{RH}$ and 12 hours of photophase was analysed using entropy $(\mathrm{H})$. Entropy $(\mathrm{H})$ was used to quantify the distribuition of deaths between ages and then quantify the impact of mortality on life expectancy. The entropy values obtained for males $(\mathrm{H}=0,245)$ and females $(\mathrm{H}=0,299)$ were intermediary between the theoretical values of $\mathrm{H}=0,5$ and $\mathrm{H}=0$ suggesting a tendency toward rectangular distribuitions in both sexes. The effect of mortality across all ages on expectation of life was different at each age. For males the highest values were found between days 10 and 20 and between days 15 and 25 for females. This findings imply that small changes in female mortality will have a greater impact on female life expectancy than will have on male expectation of life.

KEY WORDS. Diptera, Calliphoridae, Sarconesia chlorogaster, entropy, life expectation, survivorship curves
\end{abstract}

A família Calliphoridae agrupa várias espécies de importância médico-sanitária devido principalmente, aos hábitos alimentares extremamente variáveis e ao elevado grau de sinantropia que estas podem apresentar (ZUMPT 1965; GREENBERG 1971).

Sarconesia chlorogaster (Wiedemann, 1830) é um califorídeo cuja distribuição no Brasil, restringe-se apenas aos estados da Região Sul (DEAR 1979; QUEIROZ et al. 1985) e que devido a seus hábitos alimentares necrófagos (LOPES 1973), sua preferência por áreas urbanas e seu elevado grau de sinantropia (FERREIRA 1978), pode ser utilizada com indicadora do intervalo pós-morte em entomologia médicocriminal (MOURA et al. 1997).

Por ser uma espécie necrófaga, utilizando-se por conseguinte de recursos efêmeros e temporalmente irregulares, torna-se também um ótimo modelo para estudos de estrutura e dinâmica populacional. A estrutura de uma população tem sido descrita basicamente através da utilização de tabelas de vida, onde descreve-se detalhadamente a mortalidade dentro de cada idade específica (CAREY 1993). Tal procedimento é extremamente importante devido a mortalidade não ser igual em todas as idades (DEMPSTER 1975; KREBS 1994).

1) Contribuição número 1122 do Departamento de Zoologia, Universidade Federal do Paraná.

2) Departamento de Zoologia, Universidade Federal do Paraná. Caixa Postal 19020, 81531-990 Curitiba, Paraná, Brasil.

E-mail: moura@bio.ufpr.br; srbona@bio.ufpr.br

Revta bras. Zool. 16 (Supl. 1): 221 - 226, 1999 
A distribuição da mortalidade por idade pode assumir dois padrões extremos (DEMETRIUS 1978), sendo possível que todos os indivíduos morram com a mesma idade ou que os indivíduos possuam a mesma probabilidade de morrer em cada idade (CAREY 1993).

De uma maneira geral, a análise das curvas de sobrevivência tem sido feita de forma qualitativa (SILVEIRA-NETO et al. 1976). Embora o tipo de curva possa ser facilmente demonstrado, o mesmo dificilmente ocorre com a convexidade da curva, a qual é altamente sensível às condições do meio e da estrutura genética da população, fornecendo deste modo informações úteis sobre a população (DEMETRIUS 1979).

Uma das formas de se quantificar a variabilidade na distribuição da mortalidade e a convexidade da curva de sobrevivência é através da utilização do conceito de entropia (H) em demografia (DEMETRIUS 1978, 1979; GOLDMAN \& LORD 1986). O conceito de entropia foi adaptado a partir da mecânica estatística buscando medir a variabilidade da distribuição da mortalidade e também descrever o desvio da linearidade da variação do número de sobreviventes em função da idade, quantificando assim o impacto da mortalidade sobre a expectativa de vida (DEMETRIUS 1978).

Neste trabalho o objetivo foi estudar a esperança de vida dos adultos de $S$. chlorogaster, baseando-se nas características quantitativas da curva de sobrevivência, através da análise da entropia $(\mathrm{H})$.

\section{MATERIAL E MÉTODOS}

Os espécimes adultos foram coletados na região de Curitiba com armadilhas para moscas descritas por FERREIRA (1978) sendo a isca utilizada peixe em decomposição. Os adultos coletados foram acondicionados em uma gaiola $(91 \mathrm{~cm} \times 46 \mathrm{~cm}$ x $46 \mathrm{~cm}$ ) com armação de alumínio e laterais de tule. Como substrato para alimentação e postura foi fornecido peixe, sendo também ministrada uma dieta complementar composta por açúcar, levedura de cerveja e leite em pó, em iguais proporções, e água ad libitum. Os ovos obtidos a partir desta geração parental foram transferidos para um cristalizador, sendo os adultos que emergiram desta geração (F1) utilizados para o estudo ( $c f$. BONATTO 1996).

Para o estudo da longevidade dos adultos foram formados 30 casais, originários de $\mathrm{F} 1$ e pertencentes a mesma idade. Cada casal foi acondicionado em frascos de polietileno com abertura lateral coberta por tule. Como alimento e substrato de postura, foram fornecidos diariamente pedaços de peixe, dieta alimentar complementar e água ad libitum. Todos os casais foram mantidos em câmara climatizada a $27 \pm 1^{\circ} \mathrm{C}$ de temperatura, $70 \pm 10 \%$ de umidade relativa dos ar e fotofase de 12 horas (cf. BONATTO 1996).

Para o cálculo da estimativa da entropia $(\mathrm{H})$ foram utilizados os seguintes parâmetros, calculados a partir dos dados de sobrevivência em tabela de vida (CAREY 1993): $\mathbf{l}_{\mathbf{x}}$, que representa a proporção de sobreviventes a partir da emergência em relação ao intervalo $x$ de idade; $\mathbf{d}_{\mathbf{x}}=\mathbf{l}_{\mathbf{x}}-\mathbf{l}_{\mathbf{x}+\mathbf{1}}$ fornece a proporção de indivíduos que morreram entre as idades $x$ e $x+1 ; q_{\mathbf{x}}=\mathbf{d}_{\mathbf{x}} / l_{\mathbf{x}}$, a probabilidade de 
morte na idade $\mathbf{x}$ e, $\mathbf{p}_{\mathbf{x}}=\mathbf{1}-\mathbf{q}_{\mathbf{x}}$ a probabilidade de sobrevivência. A probabilidade média de sobrevivência foi calculada por $\mathbf{L}_{\mathbf{x}}=\mathbf{I}_{\mathbf{x}}-\left(\mathbf{d}_{\mathbf{x}} / \mathbf{2}\right)$; o número total de dias restantes aos sobreviventes até a idade máxima $w, \mathbf{T}_{\mathbf{x}}$, foi estimado por:

$$
\sum_{y=x}^{w} L_{y}
$$

e a esperança de vida estimada por $\mathbf{T}_{\mathbf{x}} / \mathbf{l}_{\mathbf{x}}$. Os valores da entropia $(H)$ foram calculados seguindo-se a expressão:

$$
H=\sum_{x=0}^{w} \frac{e_{x} d_{x}}{e_{0}}
$$

onde: $\boldsymbol{e}_{\boldsymbol{x}}$ representa a esperança de vida na idade $\boldsymbol{x} ; \boldsymbol{d}_{\boldsymbol{x}}$ a proporção de indivíduos que morreram entre as idades $\boldsymbol{x}$ e $\boldsymbol{x}+\mathbf{1} ; \boldsymbol{e} \boldsymbol{0}$ a esperança de vida na idade inicial e; $\boldsymbol{w}$ idade máxima alcançada pelo último indivíduo sobrevivente (CAREY 1993).

\section{RESULTADOS E DISCUSSÃO}

As curvas de sobrevivência para machos e fêmeas bem como os valores teóricos e calculados para a entropia estão demonstrados na figura 1 e os parâmetros necessários para o cálculo da entropia na tabela I.
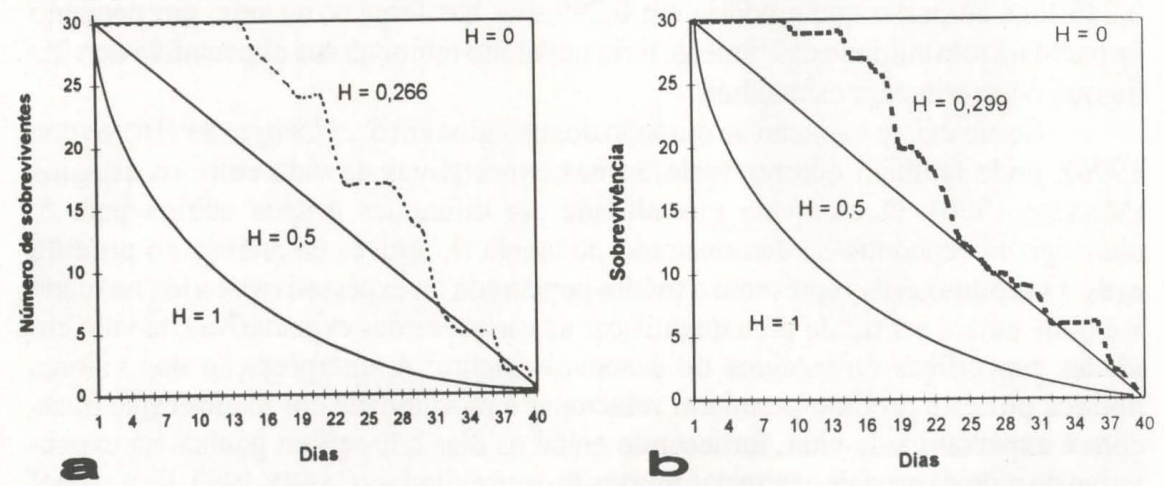

Fig. 1. Curva de sobrevivência (linha tracejada) de Sarconesia chlorogaster criada sob condições experimentais controladas, mostrando sua posição em relação aos valores teóricos da entropia (H). (a) Macho, (b) fêmea.

Tabela I. Parâmetros utilizados para o cálculo da entropia $(H)$ de machos e fêmeas de Sarconesia chlorogaster criados sob condições experimentais. (eo) Esperança de vida na idade inicial, $(w)$ idade máxima em dias alcançada pelo último indivíduo sobrevivente, $\left(\sum e_{x} d_{x}\right)$ soma das médias ponderadas das expectativas de vida em todas as idades.

\begin{tabular}{lccc}
\hline & $e_{0}$ & $w$ & $\Sigma e_{x} d_{x}$ \\
\hline Machos & 25,70 & 38 & 6,308 \\
Fêmeas & 23,86 & 39 & 7,152 \\
\hline
\end{tabular}


Quando a entropia (H) é igual a zero, obtém-se a concavidade mais extrema possível (CAREY 1993), uma vez que todos os indivíduos morrem exatamente ao mesmo tempo (VAUPEL 1986). Já para $\mathrm{H}=1$, ocorre um decréscimo geométrico na sobrevivência em relação a idade (CAREY 1993), o que corresponderia a uma probabilidade constante de mortalidade em cada idade e para $\mathrm{H}=0,5$ uma relação linear entre mortalidade e idade (DEMETRIUS 1978) (Fig. 1).

Os valores de entropia calculados para machos $(\mathrm{H}=0,245)$ e fêmeas $(\mathrm{H}=0,299)$ de Sarconesia chlorogaster, situam-se intermediariamente entre os valores teóricos de $\mathrm{H}=0$ e $\mathrm{H}=0,5$, embora haja uma tendência a $\mathrm{H}=0,5$ principalmente nas fêmeas. Os valores obtidos para $S$. chlorogaster são próximos aos valores de entropia obtidos por VON ZUBEN et al. (1996) para Chrysomya megacephala (Fabricius, 1794) ainda que, o formato geral das curvas seja diferente.

Os valor de $\mathrm{H}$ pode ser interpretado como um indicador quantitativo da concavidade da curva de sobrevivência (DEMETRIUS 1978), sendo que para $S$. chlorogaster os machos apresentam uma curva levemente mais côncava que a das fêmeas (Fig. 1), o que para C. megacephala (VON ZUBEN et al. 1996) não ocorreu. A medida de entropia também fornece indícios sobre o efeito da mortalidade na expectativa de vida, através da análise da mudança percentual produzida por uma redução de $1 \%$ na força de mortalidade em todas as idades (VAUPEL 1986). Com isso, uma redução de $1 \%$ na mortalidade, iria mudar a expectativa dos machos em 0,245 dias ao passo que mudaria em 0,299 dias nas fêmeas, ou seja, um pequeno impacto na mortalidade das fêmeas, teria um efeito maior na sua expectativa de vida do que ocorreria para os machos.

Como existe variação na duração dos estágios em S. chlorogaster (BONATTO 1996), pode também ocorrer variação nas expectativas de vida entre os estágios (MANLY 1990). O efeito da mortalidade em diferentes grupos etários para $S$. chlorogaster encontra-se demonstrado na tabela II, através da análise do produto $\mathbf{e}_{\mathbf{x}} \mathbf{d}_{\mathbf{x}}$. O produto $\mathbf{e}_{\mathbf{x}} \mathbf{d}_{\mathbf{x}}$ representa a média ponderada da expectativa de vida na idade $\mathbf{x}$ e pode então, ser usado para quantificar as variações das expectativas de vida em idades específicas ou estágios de desenvolvimento. A interpretação dos valores obtidos por este produto permitem relacionar a mortalidade em idades específicas com a expectativa de vida, fornecendo então os dias que seriam ganhos na expectativa de vida da espécie caso estas mortes fossem evitadas (CAREY 1993; GOLDMAN \& LORD 1986).

Tabela II. Valores do produto $e_{x} d_{x}$ sobre intervalos de cinco dias para machos e fêmeas de Sarconesia chlorogaster criados em condições experimentais controladas.

\begin{tabular}{ccc}
\hline Intervalo (dias) & Machos & Fêmeas \\
\hline $0-5$ & 0,000 & 0,000 \\
$5-10$ & 0,000 & 0,528 \\
$10-15$ & 2,048 & 1,099 \\
$15-20$ & 2,364 & 2,394 \\
$20-25$ & 0,170 & 2,143 \\
$25-30$ & 1,512 & 0,736 \\
$30-35$ & 0,170 & 0,150 \\
$35-40$ & 0,033 & 0,099 \\
\hline
\end{tabular}


Os resultados expressos na tabela II mostram que, para os machos de $S$. chlorogaster, aproximadamente quatro dias são perdidos devido à mortes no período compreendido entre o $10^{\circ}$ e $20^{\circ}$ dias, o que corresponde principalmente ao estádio de larva III (BONATTO 1996) e que o $17^{\circ}$ dia apresenta o maior valor de $\mathbf{e}_{\mathbf{x}} \mathbf{d}_{\mathbf{x}}$. Já para as fêmeas desta espécie, o maior valor de $\mathbf{e}_{\mathbf{x}} \mathbf{d}_{\mathbf{x}}$ ocorreu entre $015^{\circ}$ e $25^{\circ}$ dias, demonstrando que aproximadamente 4,5 dias de vida futura são perdidos pelas fêmeas nestas idades, que também correspondem principalmente ao estágio de larva III sendo que $o 13^{\circ}$ dia foi o que apresentou o maior valor individual.

Os resultados são importantes na medida em que, mesmo não havendo diferenças significativas entre as expectativas de vida de machos e fêmeas de $S$. chlorogaster (BONATTO 1996), as análises de entropia revelaram diferenças nas concavidades da curva, que, por conseguinte, podem levar a dinâmicas diferentes sendo as fêmeas, no caso de $S$. chlorogaster, as que devem sofrer os maiores impactos da mortalidade.

Sendo as fêmeas as responsáveis por encontrar os sítios de criação das larvas e tendo sua expectativa de vida mais influenciada por pequenas variações na mortalidade, a probabilidade de encontrar tais sítios seria afetada, podendo assim alterar a dinâmica do sistema (ROFF 1977; HANSKI 1987). Além de influenciar a dispersão para novos sítios de criação, a fecundidade também poderia ser afetada pois, o número de ciclos gonadotróficos é função do tempo de vida. Assim, quanto maior o tempo de vida, maior a quantidade de posturas que uma fêmea poderia realizar.

De uma maneira geral, as variações no padrão de mortalidade possuem reflexo direto na dinâmica das populações porque influenciam parâmetros importantes como a fecundidade, a qual tem papel fundamental na dinâmica de outras espécies (VON ZUBEN et. al. 1993; CONSTANTINO et. al. 1995) e a dispersão que também é característica fundamental para o sucesso em comunidades efêmeras (HANSKI 1987).

AGRADECIMENTOS. Aos Doutores Claudio Von Zuben, Emygdio L.A. Monteiro-Filho e Luciane Marinoni pela leitura crítica do manuscrito.

\section{REFERÊNCIAS BIBLIOGRÁFICAS}

BonAtTo, S.R. 1996. Ciclo de Vida de Sarconesia chlorogaster (Wiedemann) (Diptera, Calliphoridae, Toxotarsinae), criada sob condições de laboratório em dieta artificial. Revta bras. Zool. 13 (3): 685-706.

CAREY, J.R. 1993. Applied demography for biologists. New York, Oxford University Press, XVI+206p.

Constantino, R.F.; J.M. Cushing; B. Dennis \& R.A. Desharnais. 1995. Experimentally induced transitions in the behaviour of insect populations. Nature 375: $227-230$.

DEAR, J.P. 1979. A revision of the Toxotarsinae (Diptera: Calliphoridae). Pap. Avulsos Dept. Zool., São Paulo, 32 (13): 145-182.

DEMETRIUS, L. 1978. Adaptative value, entropy and survivorship curve. Nature 
275: $213-214$.

329-338.

DEMPSTER, J.P. 1975. Animal population ecology. London, Academic Press, $\mathrm{X}+155$.

FERREIRA, M.J. DE M. 1978. Sinantropia de dípteros muscoídeos de Curitiba, Paraná. I. Calliphoridae. Rev. Brasil. Biol. 38: 445-454.

Goldman, N. \& J.W. LoRD. 1986. A new look at entropy and the life table.

Demography 23: 275-282.

GREENBERG, B. 1971. Flies and disease. Ecology, classification and biotic associations. New Jersey, Princeton University Press, Vol. 1, VII+586p.

HANSKI, I. 1987. Colonization of ephemeral habitats, p. 155-185. In: A.J. GRAY;

M.J. CRAWLEY \& P.J. EDWARDS (Eds). Colonization, succession and stability. Oxford, Blackwell Scientific Publications, XI+482p.

KREBS, C.J. 1994. Ecology: The experimental analysis of distribuition and abundance. New York, Harper Collins College Publishers, XIV+801p.

LOPES, H. DE S. 1973. Collecting and rearing Sarcophagids flies (Diptera) in Brazil during forty years. An. Acad. Brasil. Ciênc. 45: 279-299.

MANLY, B.F.J. 1990. Stage-structured populations: Sampling, analysis and simulation. London, Chapman and Hall, XII+187p.

Moura, M.O.; C.J.B. DE CARvalho \& E.L.A. MonTEIRO-FILHO. 1997. A preliminary analysis of insects os medico-legal importance in Curitiba, State of Paraná. Mem. Inst. Oswaldo Cruz 92: 269-274.

QueIroz, S.M.P.; J.R. DE Almeida; C.J.B DE Carvalho \& L. Dudas. 1985. Bionomia de Sarconesia chlorogaster (Wiedemann, 1830) em Curitiba, Paraná, Brasil. An. Soc. Entomol. Bras. 14: 105-110.

RofF, D. 1977. Dispersal in dipterans: its costas and consequences. Jour. Anim. Ecol. 46: 443-456.

Silveira-Neto, S.; O. NAKANO; D. BARBIN \& N.A. Villa Nova. 1976. Manual de ecologia dos insetos. São Paulo, Editora Agronômica Ceres Ltda., 419p.

VAUPEL, J.W. 1986. How change in age-specific mortality affects life expectancy. Pop. Studies 40: 147-157.

VON ZUBEN, C.J.; S.F. DOS REIS; J.B.R. DO VAL; W.A.C. GODOY \& O.B. RiBEIRO. 1993. Dynamics of a mathematical model of Chrysomya megacephala (Diptera, Calliphoridae). Jour. Med. Entomol. 30: 443-448.

von Zuben, C.J.; W.A.C. Godoy \& E.L.A. Monteiro-FilHo. 1996. Curva de sobrevivência e estimativa de entropia em Chrysomya megacephala (Diptera: Calliphoridae). Revta bras. Ent. 40 (2): 221-224.

ZUMPT, F. 1965. Myiasis in man and animals in the Old World. London, Butterworths, 267p.

Recebido em 20.V.1998; aceito em 28.IV.1999. 